\title{
Commentary Tracheostomy decannulation: marathons and finish lines
} John E Heffner ${ }^{1,2}$

\author{
${ }^{1}$ Providence Portland Medical Center, NE Hoyt St, Portland, Oregon, 97213 USA \\ 2Oregon Health \& Science University, SW Sam Jackson Park Rd, Portland, Oregon 97239, USA
}

Corresponding author: John E Heffner, John_heffner@mac.com

Published: 31 March 2008

This article is online at http://ccforum.com/content/12/2/128

(c) 2008 BioMed Central Ltd

See related research by Stelfox et al., http://ccforum.com/content/12/2/R26
Critical Care 2008, 12:128 (doi:10.1186/cc6833)

\begin{abstract}
Critically ill patients with a tracheostomy who are recovering from respiratory failure eventually require evaluation for airway decannulation. Although expert recommendations guide decisions for managing decannulation, few if any investigative data exist to inform evidence-based care. Consequently, practice variation limits the effectiveness of weaning from tracheostomy. In an investigation reported in this issue of Critical Care, the authors surveyed experienced physicians and respiratory therapists to assess their opinions on managing airway decannulation and identified several clinical factors that they recommend for selecting patients for tracheostomy tube removal. The authors propose that these factors can assist with designing clinical trials of tracheostomy decannulation. Pending completion of such studies, this report underscores the problem of practice variation in managing tracheotomized patients after critical illness. An important implication of the study is that care providers should recognize our knowledge deficit and develop systematic protocols for improving patient care using quality improvement techniques. Such models exist in the literature for adult patients and for children with tracheostomies who are managed by expert teams with requisite knowledge and skills.
\end{abstract}

\section{Introduction}

Among the many controversies that surround tracheotomy for critically ill patients, none is of greater importance to patient outcomes - or of greater provider neglect - than management decisions to ensure tracheostomy decannulation. In this edition of Critical Care, Stelfox and colleagues [1] surveyed 309 physicians and respiratory therapists who had critical care experience and observed considerable practice variation in self-reported approaches to decannulation. Unique clinical features of patients accounted for some of this practice variation, but other variations corresponded to differing practice styles between the surveyed categories of providers, such as employment in chronic versus acute care facilities.

The investigators identified some consensus for several factors to select patients for decannulation, which included level of consciousness, cough effectiveness, amount of secretions, and oxygenation. Also, respondents defined 'decannulation failure' as the need to reintubate the airway within 48 to 96 hours after planned removal of a tracheostomy tube. As proposed by the investigators, these observations add to the findings of previous studies [2] and will help in the design of future studies to identify decision support tools for selecting patients for decannulation.

\section{Study implications for managing practice variation}

Unfortunately, however, high-quality clinical trials of tracheostomy care for critically ill patients have proven notoriously difficult to perform [3], and robust decannulation studies will not emerge any time soon. So, the more compelling interpretation of this study relates to its implications for immediately altering current clinical practice. Practice variation that corresponds to physician rather than patient differences can have one of two explanations. Clinicians may fail to adopt existing high-level scientific knowledge into their clinical practices [4]. Alternatively, their varying practices may represent differing decision-making in a setting of scientific uncertainty when no high-quality investigative findings exist. Clearly, the latter explains the observed lack of consensus for managing tracheostomy decannulation in the critically ill. Only expert-based recommendations, and not scientific knowledge, are available to guide our current practices $[5,6]$.

\section{Improving decannulation outcomes}

So how can we improve the care of tracheotomized patients in the absence of clinical trials? First, we can recognize the nature of the problem. At the outset of respiratory failure, highly skilled critical care teams manage patients in the resource intense environment of the intensive care unit (ICU). The management of tracheostomy decannulation, however, usually occurs long after transfer to non-ICU settings, where

$\mathrm{ICU}=$ intensive care unit. 
bedside care givers may lack comprehensive experience in assessing compromised airways in patients with multiple comorbidities. A failure of expertise commonly underlies a failure of decannulation.

Second, we should note that most hospitals have sufficient expert personnel to develop a team for managing decannulation care. In the pediatric treatment model, otolaryngologists have claimed tracheostomy decannulation as a core component of their specialty [7-9]. They bring to bear an arsenal of resources, including endoscopic and imaging studies for children, who usually have isolated airway disorders. In contrast, adults with complex co-morbidities after ICU care render otolaryngologists unprepared to direct management, even though these patients may have unique airway complications that require the skills of an otolaryngologist [10]. Intensivists have some of the required skills but they often do not follow patients long term outside the ICU. Unless patients are transferred to specialized ventilator weaning centers, they often recover in acute care facilities surrounded by expertise everywhere except at the bedside.

Third, we can learn from quality improvement efforts that have exploited systems of care to make up for the absence of scientific knowledge and bedside expertise. Impressive improvements in care observed recently in the ICU result from taking what we know (scientific knowledge) and what we think we know (expert consensus) and developing teambased protocols to manage ventilator weaning, sedation, central venous catheters, and other interventions, with measured outcomes used to improve the protocols. Only occasional centers have applied these quality improvement cycles to improve tracheostomy weaning [11-13]. When critical care providers and otolaryngologists cannot come regularly to the bedside, protocols make their shared expertise consistently available.

\section{Conclusion}

Stelfox and colleagues [1] have clearly defined the gaps in our tracheostomy care. We should recognize that patients with respiratory failure run a marathon toward recovery, and we should not neglect the last mile, which is of equal importance as the first 25 , if they are to cross the finish line.

\section{Competing Interests}

The author declares that they have no competing interests.

\section{References}

1. Stelfox HT, Crimi C, Berra L, Noto A, Schmidt U, Bigatello LM, Hess D: Determinants of tracheostomy decannulation: an international survey. Crit Care 2008, 12:R26.

2. Leung R, MacGregor L, Campbell D, Berkowitz RG: Decannulation and survival following tracheostomy in an intensive care unit. Ann Otol Rhinol Laryngol 2003, 112:853-858.

3. Pierson DJ: Tracheostomy and weaning. Respir Care 2005, 50: 526-533.

4. Rubenfeld GD: Implementing effective ventilator practice at the bedside. Curr Opin Crit Care 2004, 10:33-39.

5. Heffner JE: The technique of weaning from tracheostomy. Cri- teria for weaning; practical measures to prevent failure. J Crit Illness 1995, 10:729-733.

6. Heffner JE, Hess D: Tracheostomy management in the chronically ventilated patient. Clin Chest Med 2001, 22:55-69.

7. Eber E, Oberwaldner B: Tracheostomy care in the hospital. Paediatr Respir Rev 2006, 7:175-184.

8. Kontzoglou G, Petropoulos I, Noussios G, Skouras A, Benis N, Karagiannidis $\mathrm{K}$ : Decannulation in children after long-term tracheostomy. B-ENT 2006, 2:13-15.

9. Sasaki CT, Gaudet PT, Peerless A: Tracheostomy decannulation. Am J Dis Child 1978, 132:266-269.

10. Raghuraman G, Rajan S, Marzouk JK, Mullhi D, Smith FG: Is tracheal stenosis caused by percutaneous tracheostomy different from that by surgical tracheostomy? Chest 2005, 127: 879-885.

11. Lewarski JS: Long-term care of the patient with a tracheostomy. Respir Care 2005, 50:534-537.

12. Norwood MG, Spiers P, Bailiss J, Sayers RD: Evaluation of the role of a specialist tracheostomy service. From critical care to outreach and beyond. Postgrad Med J 2004, 80:478-480.

13. Doerksen K, Ladyshewsky A, Stansfield K: A comparative study of systemized vs. random tracheostomy weaning. Axone 1994, 16:5-13. 